\title{
Modelo analítico de qualidade da água acoplado com Sistema de Informação Geográfica para simulação de lançamentos com duração variada
}

\section{Analytical water quality model coupled with Geographic Information System for simulation of variable time discharges}

\section{Fernando Mainardi Fan}

Doutorando em Recursos Hídricos e Saneamento Ambiental no Instituto de Pesquisas Hidráulicas (IPH) da Universidade Federal do Rio Grande do Sul

(UFRGS) - Porto Alegre (RS), Brasil.

\author{
Walter Collischonn \\ Doutor em Recursos Hídricos e Saneamento Ambiental. Professor Adjunto do IPH da UFRGS - Porto Alegre (RS), Brasil.
}

\section{Daniel Rigo}

Doutor em Engenharia Oceânica pela Universidade Federal do Rio de Janeiro (UFRJ)- Rio de Janeiro (RJ), Brasil. Professor Adjunto da Universidade Federal do Espírito Santo (UFES) - Vitória (ES), Brasil.

\section{Resumo}

Este trabalho apresenta o desenvolvimento de um modelo de qualidade da água acoplado com um software livre de Sistema de Informação Geográfica (SIG) para representação de impactos causados por lançamentos de poluentes em grande escala, com uma visão sistêmica de toda a bacia hidrográfica e com ferramentas simplificadas para lidar com as condições típicas de baixa disponibilidade de dados. No modelo proposto, denominado SIAQUA-IPH é adotada uma metodologia baseada na solução analítica da equação de transporte de poluentes por meio de um esquema de sobreposição linear. São apresentados testes em que os resultados do modelo foram comparados com experimentos de lançamento de traçadores no Rio Paraíba do Sul, com resultados promissores, considerando-se as incertezas envolvidas.

Palavras-chave: qualidade da água; modelagem; SIAQUA-IPH.

\section{Abstract}

This paper presents the development of a water quality model coupled with an open source Geographic Information System (GIS) software representing the impacts of large scale pollutant releases, with a systemic view of the entire basin, and simplified tools to deal with typical data scarcity situations. In the proposed model, called SIAQUA-IPH, a methodology based on the pollutant transport equation analytical solution through a scheme of linear superposition is adopted. The paper also presents tests in which model results were compared to tracer releases experiments in the Paraíba do Sul river, where promising results were obtained considering the uncertainties involved.

Keywords: water quality; modeling; SIAQUA-IPH

\section{Introdução}

A modelagem matemática da qualidade da água está baseada no uso de equações que representam os processos de transporte e transformação de poluentes em corpos de água. Com a modelagem, é possível diagnosticar problemas relacionados à qualidade da água e avaliar cenários de uma forma relativamente rápida e barata (COX, 2003; BENEDINI, 2011).

Ao longo das últimas décadas, foram desenvolvidos diversos modelos de qualidade da água, que são utilizados, por exemplo, para analisar os impactos do lançamento de poluentes em trechos relativamente curtos de rios (COX, 2003; BENEDINI, 2011). 
Tipicamente, a aplicação de modelos de qualidade da água é realizada para a estimativa de impactos resultantes de lançamentos de esgotos e efluentes industriais, para o planejamento do uso de recursos hídricos em bacias hidrográficas e também para o estudo e controle de qualidade da água em lagos. Exemplos recentes dessas aplicações podem ser encontrados em Harari et al. (2013), que aplicaram modelagem para compreender a dispersão de esgotos na baía de Santos (SP), em Mariano et al. (2010), que avaliaram comportamento do efluente de uma refinaria de petróleo lançado no Rio Atibaia (Paulínia/SP), em Lins et al. (2012), que apresentaram um modelo com a finalidade de facilitar a tomada de decisões no plano de recursos hídricos da bacia do Rio Doce, em Ferreira e Cunha (2013), que estudaram o comportamento térmico do reservatório do Rio Verde (Curitiba/PR), e em Cunha et al. (2013), que simularam a hidrodinâmica e a dispersão de agentes passivos aplicados ao estudo de impacto ambiental (EIA) de um aproveitamento hidrelétrico.

Os modelos de qualidade da água existentes, em geral, representam detalhadamente uma região relativamente pequena de um corpo de água. São, portanto, modelos de escala espacial reduzida. Por outro lado, existem problemas de qualidade da água que devem ser analisados em uma escala espacial maior, abrangendo completamente bacias hidrográficas de várias dezenas de quilômetros quadrados, ou redes hidrográficas com trechos de rios de centenas a milhares de quilômetros de extensão. Exemplos de problemas desse tipo são os lançamentos acidentais de substâncias perigosas, como no rompimento de barragens de rejeitos de mineração, ou em acidentes com caminhões que transportam cargas perigosas.

Outro tipo de problema que envolve uma escala espacial maior são os planos de bacia hidrográfica, em que é necessário dispor de modelos capazes de representar toda uma bacia hidrográfica da ordem de algumas dezenas de quilômetros quadrados, com relativamente poucos dados. Nesse tipo de problema, normalmente há diversos pontos de lançamento de poluentes, e é necessário obter resultados em diferentes cenários de desenvolvimento em muitos pontos de interesse.

Uma importante questão relacionada à escala é que os impactos sobre a qualidade da água podem ocorrer a grande distância do ponto de lançamento do poluente. Por exemplo, no acidente do rompimento da barragem de rejeitos na cidade de Cataguases (zona da mata de Minas Gerais), em março de 2003, a poluição atingiu o córrego Cágados e depois o Rio Pomba, em Minas Gerais, que, por sua vez, contaminou o Rio Paraíba do Sul, no Rio de Janeiro, chegando até ao mar (GONÇALVES et al., 2007).

Além disso, como foi o exemplo do caso citado, o ponto de lançamento pode estar localizado em um pequeno afluente de um rio maior e, por esse motivo, não basta simular detalhadamente apenas o rio principal em um modelo matemático, mas é necessário representar, ainda que de forma simplificada, toda a rede de drenagem da bacia.
Um fator que dificulta a aplicação de modelos de qualidade da água em grande escala é que, tipicamente, não há dados nem recursos para desenvolver um modelo detalhado de todo o sistema. Por exemplo, não há informações detalhadas sobre a quantidade ou concentração do material lançado. A vazão em cada trecho de rio ou em cada seção transversal não é perfeitamente conhecida. Da mesma forma, em grande escala não estão disponíveis, em geral, os dados necessários para caracterizar as seções transversais ou características geométricas do rio, que permitam calcular com precisão a velocidade da água.

Nesses problemas de grande escala, pode ser especialmente interessante a integração dos modelos de qualidade da água com Sistemas de Informação Geográfica (SIG)

A integração da modelagem de qualidade da água com SIG pode ser especialmente útil quando os locais onde vão ocorrer todos os lançamentos de poluentes em uma bacia hidrográfica não são conhecidos a priori. Isso é especialmente importante no caso do lançamento de poluentes por descargas acidentais, como a queda de um caminhão com carga perigosa em um rio, ou o rompimento de uma barragem de rejeitos. Da mesma forma, em alguns casos não está claro antes do início dos trabalhos exatamente onde são desejados os resultados, ou os resultados das simulações de qualidade da água são desejados em muitos lugares diferentes.

A integração de modelos de qualidade da água com SIG é um campo que tem se desenvolvido nas últimas décadas, a partir de trabalhos como o de Hession e Shanholtz (1988), Olivieri et al. (1991), João e Walsh (1992) e Tim et al. (1992), que utilizaram plataformas SIG para obter dados de entrada para a modelagem. Posteriormente, surgiram trabalhos de integração com modelos existentes, sendo utilizados a partir de interfaces dentro de plataformas de SIG, como apresentado por Srinivasan e Arnold (1994), USEPA (2003) e USACE (2003). Atualmente, a tendência é o desenvolvimento de aplicações completamente integradas com SIG, como nos trabalhos de Foster e McDonald (2000), Strager et al. (2010), Lins et al. (2012) e Ferrer et al. (2012), que desenvolveram modelos e métodos acoplados à estrutura de plataformas SIG

O presente trabalho apresenta um modelo de qualidade da água desenvolvido para simular os impactos do lançamento de efluentes em escala de bacias hidrográficas da ordem de várias dezenas de quilômetros quadrados, em situações com relativa carência de dados. O modelo proposto se distingue de propostas anteriores, porque utiliza uma técnica de simulação baseada em soluções analíticas da equação de dispersão aplicada trecho a trecho de uma rede de drenagem vetorial, já que é aplicável em grandes bacias onde os pontos de lançamento de efluentes e os pontos de interesses não são conhecidos a priori e opera de forma completamente integrada com um Sistema de Informação Geográfica, de acordo com as tendências mais atuais de acoplamento. O modelo foi denominado SIAQUA-IPH, que é um acrônimo para Simulador Analítico de Qualidade da Água, seguido da sigla do Instituto de Pesquisas Hidráulicas, onde a pesquisa foi desenvolvida. 


\section{Metodologia}

A metodologia do modelo SIAQUA-IPH envolve cinco etapas: (i) o pré-processamento de dados geoespaciais; (ii) a definição de atributos hidráulicos; (iii) a inclusão de lançamentos; (iv) o cálculo de propagação; e (v) o pós-processamento para visualização dos resultados.

\section{Pré-processamento de dados geoespaciais}

No modelo SIAQUA-IPH, a rede hidrográfica da bacia é representada por trechos de rios conectados, como mostra a Figura 1. A definição da rede hidrográfica é realizada em uma etapa anterior à aplicação do modelo propriamente dito, utilizando ferramentas de geoprocessamento aplicado aos recursos hídricos, como as ferramentas ArcHydro Tools (MAIDMENT, 2002), ou o conjunto TauDEM Tools (TARBOTON, 2002).

$\mathrm{Na}$ etapa de pré-processamento, é gerada a rede de drenagem da bacia hidrográfica a partir da análise de um Modelo Digital de Elevação (MDE) da região da bacia, conforme descrito em Buarque et al. (2009).

A rede de drenagem é composta por dois tipos de trechos de rio: trechos incrementais e trechos de cabeceira. Um trecho de rio incremental é definido como a extensão de rio entre duas confluências. Um trecho de rio de cabeceira é definido como a extensão do curso de água desde a nascente até a primeira confluência (ver Figura 1 com exemplo de rede de drenagem). A posição das nascentes é definida por um critério relacionado à área de drenagem mínima, a partir da qual ocorre o escoamento concentrado (FAN et al., 2013). Uma descrição mais aprofundada das ferramentas de pré-processamento pode ser encontrada em Fan (2011).

Foi adotado no modelo SIAQUA-IPH, do ponto de vista hidrológico, regime permanente de vazões, isto é, as vazões nos trechos de rios não se alteram ao longo do tempo. Também é adotada a hipótese de escoamento unidimensional, com mistura completa e instantânea dos poluentes lançados, e a seção da calha dos rios é considerada retangular.

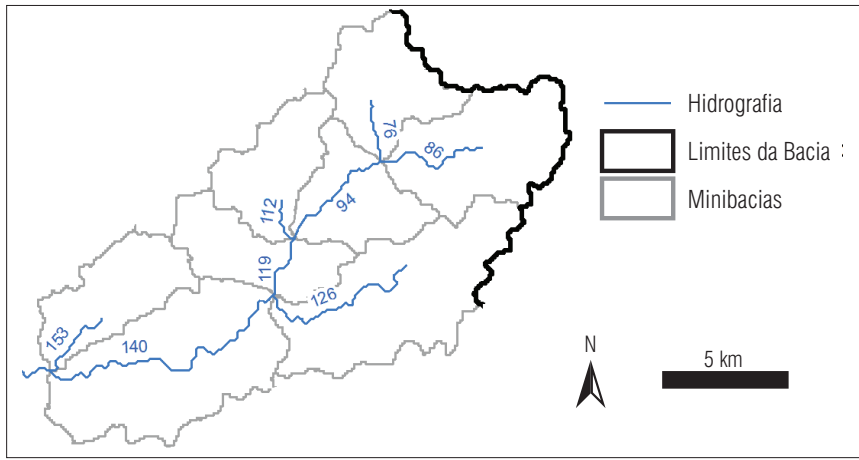

Figura 1 - Esquema de representação de uma bacia hidrográfica em pequenas minibacias, cada uma contendo um trecho de rio.
Cada trecho de rio tem um código identificador e mais sete outras características, ou atributos, necessários para os cálculos de propagação dos poluentes: o comprimento, a declividade, a largura da calha, a área de drenagem, a vazão, a velocidade média da água e o código identificador do trecho de rio localizado a jusante.

O código identificador de um trecho de rio é um número arbitrário associado ao trecho de rio durante o pré-processamento. Um atributo importante de um trecho de rio é o código do trecho localizado a jusante, que define a conexão das partes que compõem a rede de drenagem. Esse atributo é definido automaticamente na etapa de pré-processamento, utilizando-se ferramentas como o ArcHydro Tools (MAIDMENT, 2002). A Figura 1 apresenta um exemplo simplificado da rede de drenagem com os trechos de rio codificados. No exemplo da Figura 1, o trecho de rio 94 está localizado a montante do trecho 119; portanto, a água e os poluentes do trecho 94 seguem para o trecho 119

O comprimento do trecho de rio, a área de drenagem e a declividade são calculados por um algoritmo que percorre as células do MDE de forma semelhante à apresentada por Paz e Collischonn (2008). Esse cálculo também é realizado durante o pré-processamento de forma automática.

\section{Definição de atributos hidráulicos}

A largura da calha é obtida a partir de uma relação geomorfológica entre a largura do rio ( $B_{\text {rio }}$ em metros) e a área de drenagem da bacia no final do trecho de rio considerado $\left(A_{\text {dren }}\right.$ em quilômetros quadrados). Relações geomorfológicas podem ser elaboradas a partir de dados de largura em seções transversais de postos fluviométricos, por exemplo. Esse método é geralmente válido para bacias hidrográficas com características físicas homogêneas. Dois exemplos de trabalhos nos quais foram aplicadas relações geomorfológicas de forma satisfatória para estudos hidrológicos podem ser encontrados em Collischonn (2001), que ajustou a curva $B_{\text {rio }}=3,2466$. $\mathrm{A}_{\text {dren }}{ }^{0,4106}$ para a bacia dos rios Taquari-Antas (RS), e em Paiva (2009), que ajustou a curva $B_{\text {rio }}=0,8054 \cdot A_{\text {dren }}{ }^{0,5289}$ para a bacia do Rio Amazonas. No SIAQUA-IPH, os parâmetros da curva podem ser ajustados, bem como os valores calculados por ela podem ser editados manualmente.

A vazão de cada trecho de rio pode ser obtida de duas formas: regionalização ou leitura de um arquivo de dados. No caso da regionalização, a vazão de cada trecho do rio é relacionada à área de drenagem usando-se uma equação de regionalização desenvolvida previamente. Tipicamente seria utilizada uma equação de regionalização que permitiria estimar uma vazão de referência, como a Q90, a partir da área de drenagem da bacia. Como a área de drenagem da bacia é diferente em cada trecho do rio, a vazão Q90 também é diferente e cresce de montante para jusante. 
Outra forma de definir a vazão de cada trecho do rio é a leitura de um arquivo de dados. O modelo SIAQUA, descrito aqui, compartilha o mesmo tipo de discretização, em pequenas sub-bacias e trechos de rio, adotado no modelo hidrológico chuva-vazão de grandes bacias MGB-IPH (COLLISCHONN \& TUCCI, 2001; FAN, 2011). No modelo MGB-IPH, foram incluídas rotinas de cálculo de vazões de referência para cada trecho de rio, que geram as vazões $Q_{90}, Q_{70}, Q_{50}, Q_{30}$ e $Q_{10}$, que são as vazões com probabilidade de excedência de 90 a 10\%. A leitura desse arquivo no modelo de qualidade da água SIAQUA permite selecionar cenários de vazão mediana $\left(Q_{50}\right)$, vazão baixa $\left(Q_{90}\right)$ ou vazão alta $\left(Q_{10}\right)$. O cenário escolhido é adotado para toda a bacia, ou seja, se é selecionado o cenário da $\mathrm{Q}_{90}$, então se considera que cada um dos trechos tem a vazão igual à sua respectiva $Q_{90}$.

A velocidade média da água é calculada automaticamente admitindo válida a equação de Manning, a partir da vazão definida para cada trecho de rio, e considerando que o escoamento ocorre em regime permanente e uniforme, em uma seção transversal retangular, e que o raio hidráulico é igual à profundidade média, o que é uma aproximação válida na maior parte das situações em rios naturais.

\section{Inclusão de lançamentos de poluentes}

De acordo com o tempo de duração do lançamento de poluente, os tipos de lançamentos que podem ser simulados no modelo são: a) contínuo e permanente; b) instantâneo; c) transiente com duração limitada. A Figura 2 apresenta esses três tipos de lançamentos.

O tipo de lançamento contínuo e permanente é aquele que ocorre de forma contínua, sem alterações de quantidade e concentração ao longo do tempo. O tipo de lançamento instantâneo é o que ocorre quando uma carga de poluentes é despejada de forma súbita, ao longo de um período de tempo muito pequeno. $\mathrm{O}$ tipo de lançamento transiente com duração limitada é aquele que tem início em um instante de tempo e uma duração finita ao longo da qual a quantidade e a concentração do poluente são constantes, tendo um final em um instante de tempo posterior $\left(\mathrm{t}_{2}\right)$.

Os lançamentos de poluentes que ocorrem na bacia hidrográfica são inseridos no modelo de qualidade da água por meio dos mapas na janela principal do SIG, clicando sobre os trechos de rio no mapa e informando o tipo de lançamento e as demais características, como concentração, duração temporal ou carga. A partir de então, são realizados os cálculos de propagação de poluentes.

\section{Cálculos de propagação de poluentes}

Os cálculos do transporte de poluentes ao longo da rede de drenagem são realizados trecho a trecho, utilizando-se soluções analíticas da equação de transporte.

Considerando-se um lançamento instantâneo de uma massa M de um poluente em um ponto $\mathrm{x}=0 \mathrm{em}$ um rio, a concentração em qualquer ponto x localizado a jusante, onde x é a distância em metros a partir do ponto de lançamento, e em um instante t, a partir do momento do lançamento, pode ser calculada pela Equação 1 (CHAPRA, 1997; CHANSON, 2004; DEVENS et al., 2006; RIBEIRO et al., 2011):

$c(x, t)=\frac{M}{A \cdot \sqrt{4 \cdot \pi \cdot E_{L} \cdot t}} \cdot \exp \left[\left(-(x-u \cdot t)^{2} / 4 \cdot E_{L} \cdot t\right)-k \cdot t\right]$

onde:

$\mathrm{c}(\mathrm{x}, \mathrm{t})$ : concentração, em mg. $\mathrm{L}^{-1}$, em um ponto distante $\mathrm{x}$ metros do ponto de lançamento e em um instante de tempo t segundos a partir do instante de lançamento;

$E_{L}$ : coeficiente de dispersão longitudinal $\left(\mathrm{m}^{2} \cdot \mathrm{s}^{-1}\right)$;

$u$ : velocidade média do escoamento no sentido longitudinal ( $\mathrm{m} . \mathrm{s}^{-1}$ );

M: massa injetada no sistema (g);

A: área da seção transversal onde ocorre a dispersão longitudinal $\left(\mathrm{m}^{2}\right)$; $k$ : coeficiente de decaimento ambiental de primeira ordem do constituinte $\left(\mathrm{s}^{-1}\right)$.

Considerando-se um exemplo em que ocorre um lançamento com massa $\mathrm{M}_{0}$ (em g) no trecho de rio 86 (Figura 1), o cálculo da dispersão do poluente nesse trecho de lançamento é realizado usando-se a Equação 1. O polutograma é calculado para a posição $x_{f}$, que corresponde ao final do trecho em que ocorre o lançamento, para todos os valores de t entre zero e $t_{d}$, onde $t_{d}$ é um tempo suficientemente longo para permitir representar o trânsito dos poluentes por toda a bacia, até a exutória. A Figura 3B e C ilustra o processo de cálculo do transporte usando-se a Equação 1, desde o ponto de lançamento até o final do trecho.

Seguindo-se com o exemplo conforme a Figura 3, no final do trecho 86, há uma confluência entre os trechos 86 e 76, dando origem ao trecho de código 94. No local da confluência, considera-se que ocorre mistura completa e imediata da água. Assim, a concentração no início do trecho 94 pode ser calculada considerando-se

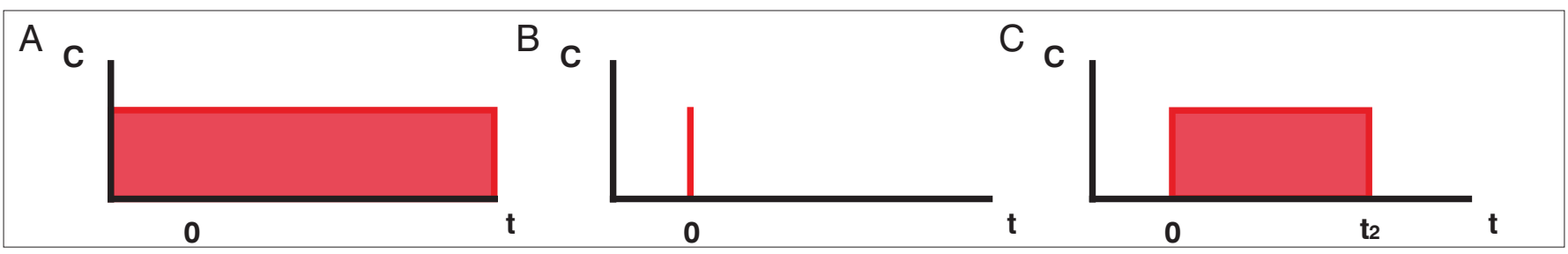

Figura 2 - Tipos de lançamentos. (A) contínuos e permanentes; (B) instantâneos; e (C) transiente com duração limitada. 
um processo simples de diluição, de acordo com a relação entre as vazões dos trechos 86 e 76

O cálculo a ser feito para a obtenção do polutograma dos demais trechos de rio, como para o trecho 119, não pode ser feito da mesma forma apresentada para os dois trechos a montante (86 e 94), uma vez que a informação de entrada da solução analítica da equação de advecção-dispersão é a quantidade de massa despejada no trecho ( $\mathrm{M}_{0}$ em $\mathrm{g}$ ), e a informação disponível, advinda de montante, é um perfil de concentrações espalhadas no tempo, em unidades de concentração (em mg. $\mathrm{L}^{-1}$ ).

Para tornar possível esse cálculo de dispersão, adotou-se no modelo uma estratégia de discretização em um número finito de partes para o polutograma do início do trecho 94 (para cada passo de tempo de cálculo, é definida uma parcela de massa), conforme mostrado na Figura 4.

Essas pequenas parcelas, que constituem o polutograma de entrada do trecho de rio 94, são multiplicadas pela vazão do mesmo, convertendo as mesmas para unidades de massa distribuídas no tempo. Essas unidades são, então, consideradas lançamentos instantâneos individuais no curso de água e, portanto, são utilizadas como despejos que ocorrem temporalmente distribuídos no trecho de rio, possibilitando a aplicação da solução analítica da equação de advecção-dispersão longitudinal. O conjunto de polutogramas individuais resultantes pode ser posteriormente somado para a obtenção do polutograma resultante ao final do trecho de rio 94. Esse processo completo é mostrado na Figura 5B, C e D.

Para o cálculo da concentração de poluentes no trecho de rio 119, a jusante do trecho 94, após a soma de polutogramas, o modelo ainda faz a diluição das concentrações calculadas com a vazão incremental da minibacia 94 e com a vazão proveniente do trecho de rio 112. A Figura 5 também apresenta de forma simplificada o cálculo das concentrações no trecho 119.

A mesma metodologia é aplicada sucessivamente para os demais trechos de rio a jusante, até que o exutório da bacia seja alcançado. O resultado gerado por todo o processo constitui-se no polutograma da contaminação que viaja pelo rio em todos os trechos, visualizado por um observador parado em um ponto a jusante do despejo. Esse observador equivale a uma captação de água, por exemplo.

Esse processo de discretização e propagação dos lançamentos individuais consiste no principal princípio adotado no SIAQUA-IPH para cálculo da propagação e dispersão de poluentes. Por se assemelhar com a técnica utilizada em hidrologia de soma de hidrogramas

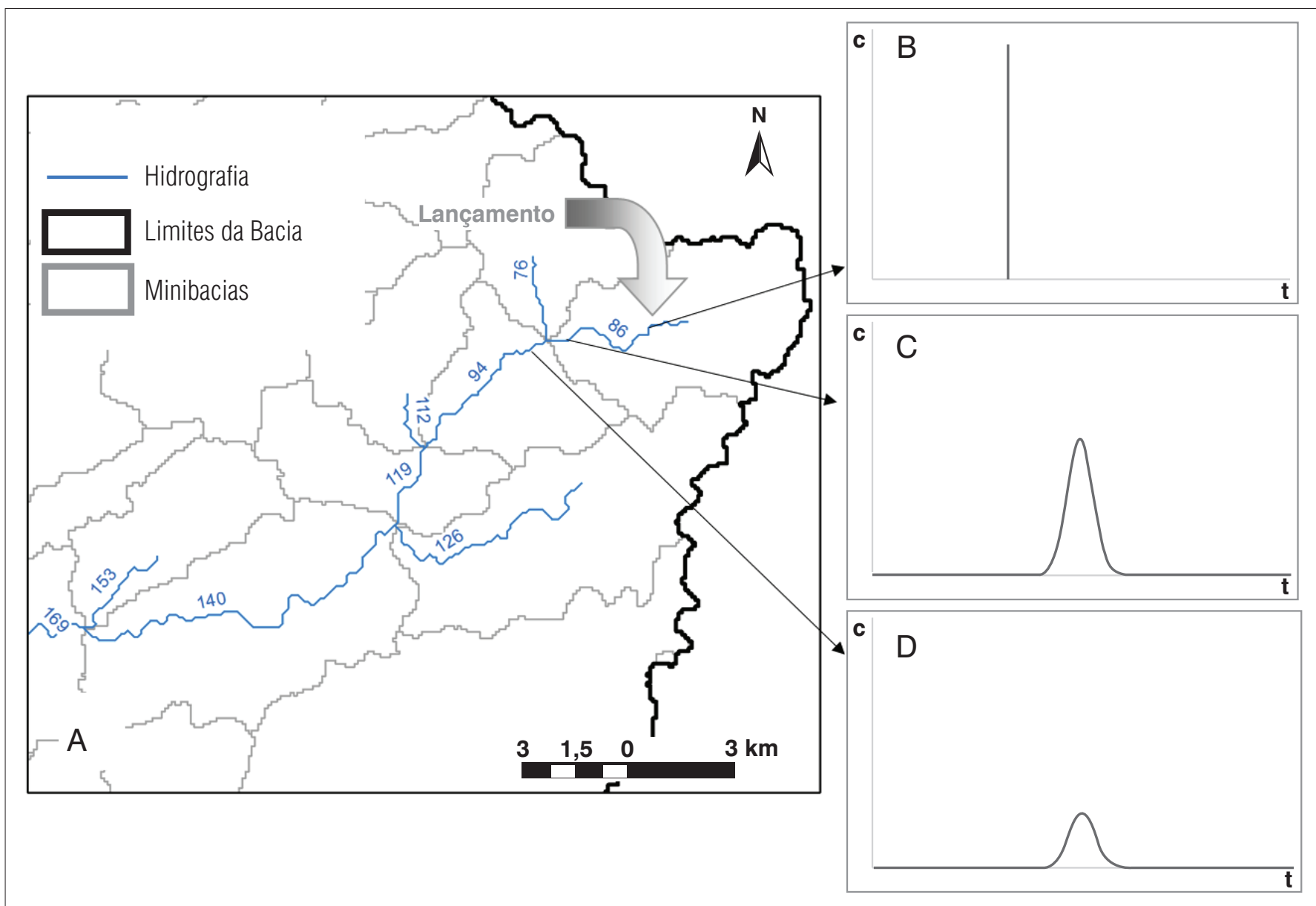

Figura 3 - Processo de cálculo de concentrações no trecho a jusante do trecho de lançamento. (A) Mapa da região; (B) Polutograma no ponto de lançamento no trecho 86; (C) Polutograma no final do trecho 86, após a propagação; (D) Polutograma no início do trecho 94 após diluição pela vazão proveniente do trecho 76 . 


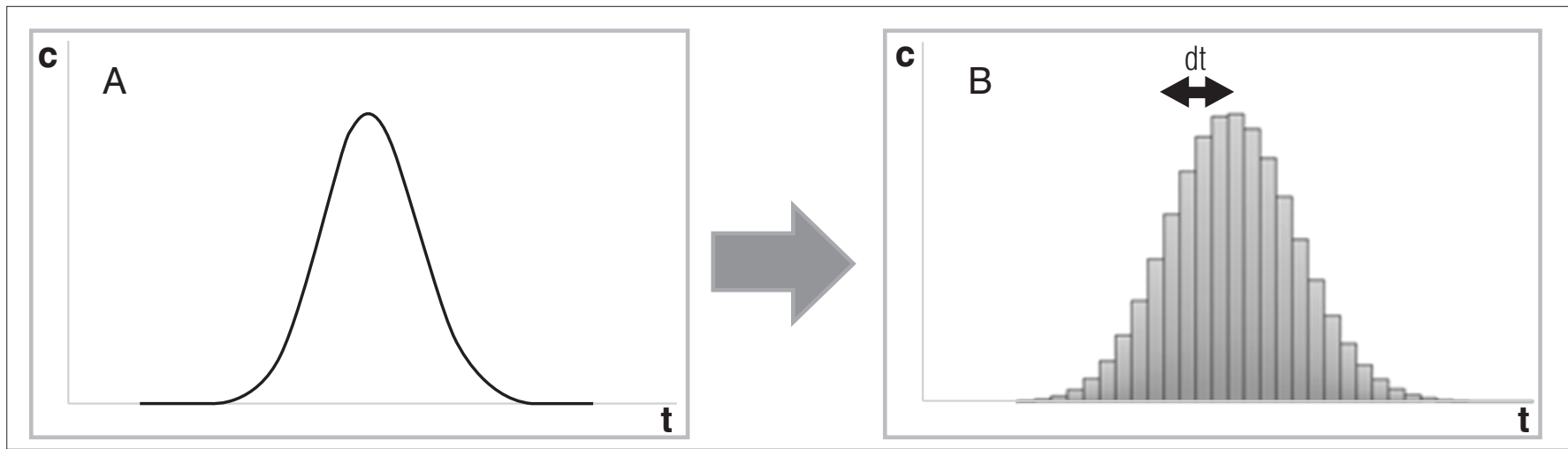

Figura 4 - Discretização do polutograma de entrada de um trecho de rio. (A) Polutograma no início do trecho de rio 94; (B) Polutograma no início do trecho de rio 94 discretizado.

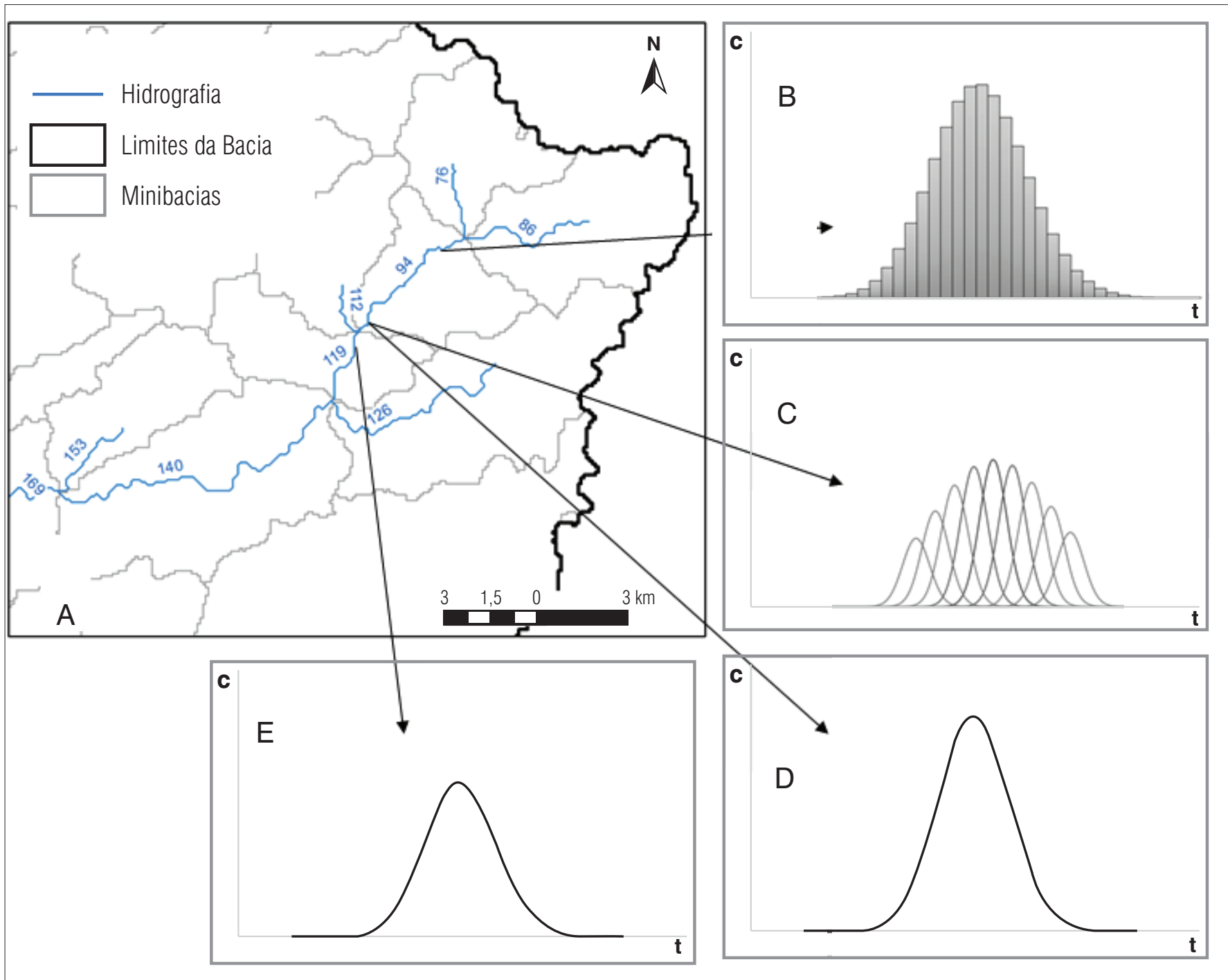

Figura 5 - Resumo dos passos de cálculo das concentrações no trecho 119. (A) Mapa da região; (B) Polutograma no início do trecho 94 após discretização; (C) Polutogramas resultantes ao final do trecho 94, após propagação, usando-se a equação da advecção-dispersão longitudinal dos lançamentos discretizados; (D) Polutograma no final do trecho 94 após soma dos polutogramas resultantes da propagação de cada um dos lançamentos discretizados; (E) Polutograma no início do trecho 119 após diluição, considerando-se a entrada de vazão do trecho 112. 
unitários (convolução do hidrograma unitário), essa técnica pode ser denominada de Convolução do Polutograma (CHANSON, 2004; RIBEIRO et al., 2011).

Segundo Chanson (2004), como a equação diferencial é linear, é possível utilizar esse princípio da sobreposição para estimar o efeito combinado de uma série de diferentes lançamentos. Entre outras experiências disponíveis, Kilpatrick e Cobb (1985) e Ribeiro et al. (2011) também utilizam esse princípio. Contudo, geralmente a soma de polutogramas é usada para a representação apenas de efeitos da soma de vários lançamentos, e não para a representação de confluências em redes hidrográficas, o que é adotado no SIAQUA-IPH para o uso de equações analíticas na simulação de toda uma bacia hidrográfica.Para lançamentos com outras durações, adotou-se a mesma metodologia de cálculo usando-se convolução de polutogramas. No entanto, são usadas as soluções analíticas específicas para durações intermitentes e contínuas, que são dadas por O'Loughlin e Bowmer (1975) e Runkel (1996):

$\left.c(x, t)=\frac{c_{0}}{2}\left[e^{\frac{u x(1-\Gamma)}{2 E_{L} .} \operatorname{erfc}}\left((x-u . t . \Gamma) / 2 \sqrt{E_{L} . t}.\right)+e^{\frac{-u x(1+\Gamma)}{2 E_{L}} \cdot \operatorname{erfc}(}(x+u . t . \Gamma) / 2 \sqrt{E_{L} . t}\right)\right]$

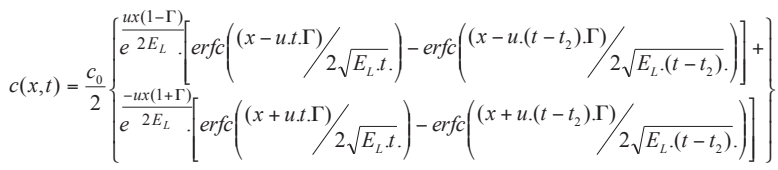

$\Gamma=\sqrt{1+4 \eta}$ $\eta=\frac{k \cdot E_{L}}{u^{2}}$

onde:

$\mathrm{c}(\mathrm{x}, \mathrm{t})$ : concentração no local x no instante de tempo t (mg..-1-1);

$c_{0}$ : concentração do lançamento no curso de água (mg.L-1-1);

$E_{L}$ : coeficiente de dispersão longitudinal $\left(\mathrm{m}^{2} \cdot \mathrm{s}^{-1}\right)$;

$t$ : instante de tempo em que se deseja saber a concentração (s);

$t_{2}$ : instante de tempo em que se encerra o lançamento transiente (s);

$x$ : local onde se deseja saber a concentração (m);

$u$ : velocidade média do escoamento no sentido longitudinal $\left(\mathrm{m} \cdot \mathrm{s}^{-1}\right)$;

M: massa injetada no sistema (g);

A: área da seção transversal onde ocorre a dispersão longitudinal $\left(\mathrm{m}^{2}\right)$; $k$ : coeficiente de decaimento ambiental do constituinte $\left(\mathrm{s}^{-1}\right)$;

$\operatorname{erfc}(x)$ : função erro complementar.

A Equação 2 é usada quando té menor ou igual a t 2 e a Equação 5, quando té maior que $t_{2}$.

Além disso, no caso da simulação de lançamentos permanentes (nos quais não há dispersão) de substâncias comumente encontradas em esgotos (demanda bioquímica de oxigênio DBO, oxigênio dissolvido - OD, nitrogênio, fósforo e coliformes), foi acoplado ao modelo o uso de equações conhecidas de sequências de reações de primeira ordem, como as apresentadas por Chapra (1997), para a simulação de todas as fases diferentes dos constituintes.

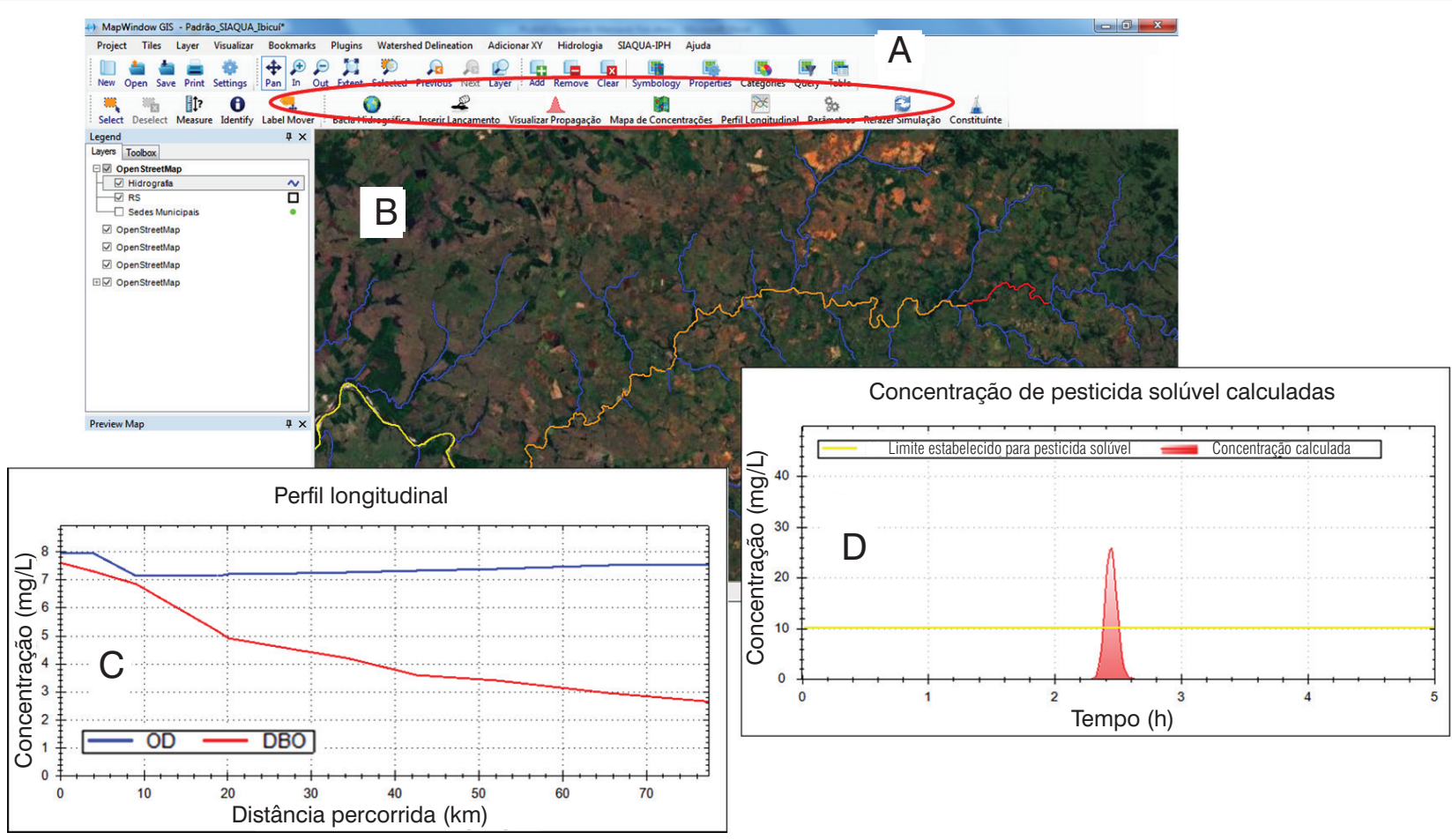

Figura 6 - Visão geral das ferramentas da integração com Sistema de Informação Geográfica desenvolvidas. 


\section{Implementação computacional integrada com Sistema de Informação Geográfica}

O modelo SIAQUA foi implementado por meio da programação de um código suplementar, também conhecido como "plugin", que é adicionado ao software de SIG MapWindow GIS ${ }^{\circledR}$ (Ames et al., 2008). O MapWindow GIS ${ }^{\circledR}$ é um programa de código aberto que pode ser obtido gratuitamente na internet e conta com um conjunto de ferramentas básicas de SIG, permitindo que sejam desenvolvidas ferramentas adicionais em linguagem VB.Net.

A Figura 6 apresenta uma visão geral das ferramentas de integração com SIG desenvolvidas para o SIAQUA-IPH. No menu superior, em A, é dado destaque para o menu de ferramentas do SIAQUA-IPH. Na janela central de mapas, em B, é mostrado um mapa de cores simbolizando o grau de impacto de um lançamento de poluentes. Também são apresentados dois gráficos que correspondem a um perfil longitudinal de DBO-OD, em C, e um polutograma de um lançamento instantâneo, em D.

\section{Estimativa de coeficientes e calibração do modelo}

Um parâmetro importante para a simulação da dispersão de contaminantes é o coeficiente de dispersão longitudinal, $\mathrm{E}_{\mathrm{L}}$, apresentado na Equação 1. Adotou-se para o SIAQUA-IPH que o coeficiente de dispersão longitudinal é definido de forma distribuída, para cada um dos trechos de rio que compõem a bacia. Isso permite que a diversidade de características físicas que compõem um curso de água seja considerada no modelo.

Por padrão, $\mathrm{o} \mathrm{E}_{\mathrm{L}}$ de cada um dos trechos de rio que compõem a bacia simulada é estimado inicialmente utilizando-se uma regra de decisão com base nas características físicas de cada trecho e na faixa de aplicabilidade de equações empíricas adicionadas ao modelo. Com a adoção desse método padronizado de seleção de equações empíricas, buscou-se diminuir a incerteza da aplicação do modelo em relação à escolha da equação por usuários. Todavia, as equações também podem ser selecionadas manualmente no modelo, ou o valor de coeficiente de dispersão dado como entrada. Os trabalhos de Devens e Barbosa Júnior (2010) e de Bowie et al. (1985) trazem uma revisão ampla sobre as diversas equações empíricas existentes para a determinação de $\mathrm{E}_{\mathrm{L}}$, que foram incorporadas ao SIAQUA-IPH considerando-se os seus respectivos limites de aplicabilidade.

Além do coeficiente $\mathrm{E}_{\mathrm{L}}$, todos os outros parâmetros do modelo podem ser alterados. Esses parâmetros seriam os coeficientes cinéticos dos lançamentos efetuados e as informações físicas dos trechos de rio, como a velocidade da água e o formato da calha do rio.

\section{Estudo de caso}

Como teste para o SIAQUA-IPH, foi realizada a avaliação dos resultados do modelo em comparação com concentrações observadas em um trabalho de campo (RIGO, 1992). O objetivo dos testes foi averiguar os erros cometidos em condições pretendidas para a aplicação do SIAQUAIPH, nas quais geralmente se tem poucos dados detalhados disponíveis.

Para tanto, procurou-se reproduzir as concentrações observadas em um teste com traçadores na bacia do Rio Paraíba do Sul. De uma forma geral, os ensaios consistiram no lançamento de uma quantidade conhecida do traçador Uranina (Fluoresceína Sódica) em um ponto denominado $\mathrm{SO}$ e no posterior monitoramento das curvas de passagem em outros pontos a jusante (S1 até S6). Esses pontos de monitoramento são ilustrados na Figura 7 e os dados dos lançamentos são apresentados na Tabela 1. A descrição detalhada e os dados originais dos ensaios de campo podem ser encontrados no trabalho de Rigo (1992).

A seguir, são apresentados os resultados de aplicação do SIAQUAIPH para reprodução desses lançamentos, incluindo o uso de todas as soluções desenvolvidas para preparação dos dados de entrada e os polutogramas das simulações. É importante destacar que não foi identificada mistura completa dos constituintes no curso de água no ponto S1, a cerca de 3,6 km do local de lançamento. Desta forma, não são esperadas boas representações das plumas observadas nesse ponto.

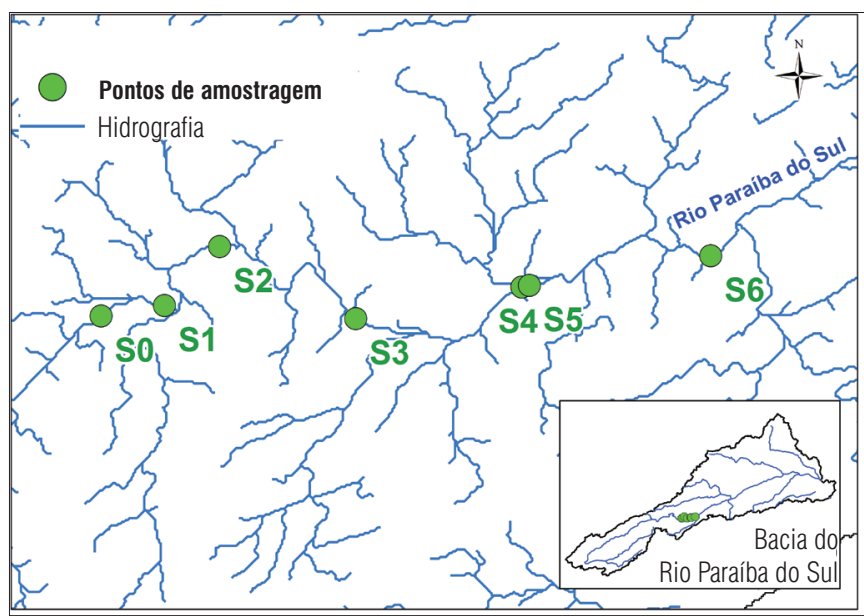

Figura 7 - Pontos de monitoramento (S0 até S6) no Rio Paraíba do Sul. Adaptado de Rigo (1992).

Tabela 1 - Detalhes dos lançamentos.

\begin{tabular}{|lccc|}
\hline $\begin{array}{l}\text { Ponto, hora e data } \\
\text { da injeção }\end{array}$ & Localização & $\begin{array}{c}\text { Vazão } \\
\left(\mathrm{m}^{3} \cdot \mathrm{s}^{-1}\right)\end{array}$ & $\begin{array}{c}\text { Massa } \\
(\mathrm{kg})\end{array}$ \\
\hline S0; 6h35; 09/09/86 & Rio Paraíba do Sul & 272 & 10,0 \\
\hline S0; 6h15; 08/01/87 & Rio Paraíba do Sul & 342 & 5,0 \\
\hline S0; 7h00; 29/01/87 & Rio Paraíba do Sul & 639 & 5,0 \\
\hline S0; 7h00; 15/12/87 & Rio Paraíba do Sul & 294 & 5,0
\end{tabular}

Fonte: Adaptado de Rigo (1992). 


\section{Resultados de teste do modelo}

\section{Preparação dos dados de entrada utilizando Sistema de Informação Geográfica}

Para a representação dos eventos para os quais se têm dados observados no Rio Paraíba do Sul, optou-se pela discretização automática da bacia por meio do pacote TauDEM Tools, disponível no MapWindow GIS ${ }^{\circledR}$. A área total da bacia do Rio Paraíba do Sul é de aproximadamente $55.400 \mathrm{~km}^{2}$. Com a aplicação do conjunto de ferramentas, ela foi dividida em 13.878 trechos de rio, com um comprimento médio de trecho de rio de $2 \mathrm{~km}$. Após essa discretização da bacia, o arquivo de entrada para o modelo de qualidade da água foi preparado utilizando-se as ferramentas desenvolvidas acopladas com o SIG.

Os dados hidráulicos de entrada do SIAQUA-IPH para o estudo de caso apresentado foram obtidos pelo uso do modelo MGB-IPH na bacia. O modelo hidrológico foi calibrado para a região de estudo utilizando-se a informação de estações fluviométricas. O resultado mais detalhado da divisão da rede hidrográfica da região em trechos de rio e a localização dos postos fluviométricos usados são apresentados na Figura 8.

Todos os dados de entrada preparados foram adicionados ao modelo usando-se as soluções de acoplamento desenvolvidas e, posteriormente, os lançamentos foram inseridos na rede hidrográfica por meio do SIG.

\section{Simulação dos eventos}

Os eventos relatados foram simulados utilizando-se os dados de entrada preparados para o SIAQUA-IPH. Como exemplo, os polutogramas obtidos para o ensaio do dia 08/01/1987 são apresentados na Figura 9.

Os resultados em S1 são apresentados nas figuras somente como referência (neste ponto não existe mistura completa). $\mathrm{Na}$ Tabela 2, é apresentado um resumo das diferenças absolutas e dos erros percentuais entre as concentrações de pico e tempos de pico encontrados entre as plumas observadas e as simuladas para os pontos onde se têm dados observados. Os erros percentuais foram

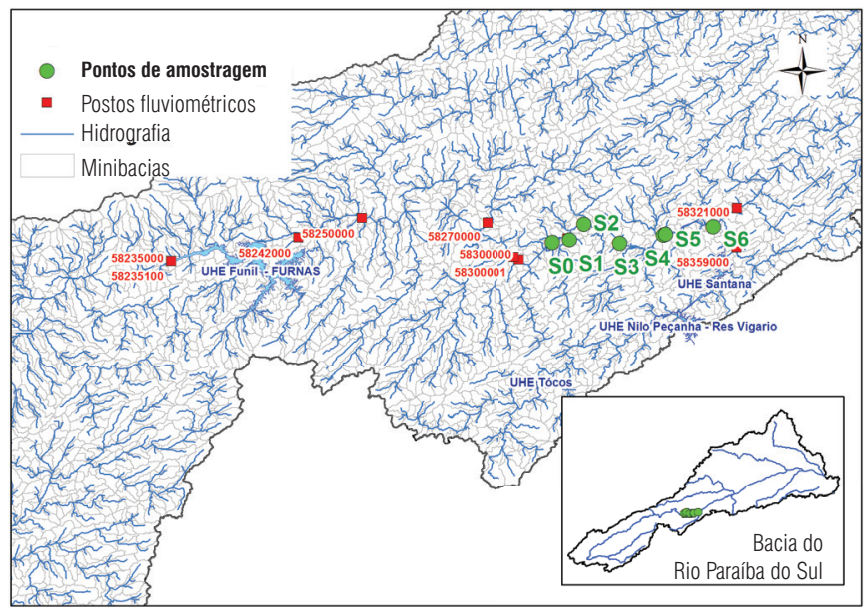

Figura 8 - Detalhe da divisão da rede hidrográfica da região e a localização dos postos fluviométricos utilizados. S0 até S6: pontos de amostragem de traçador.

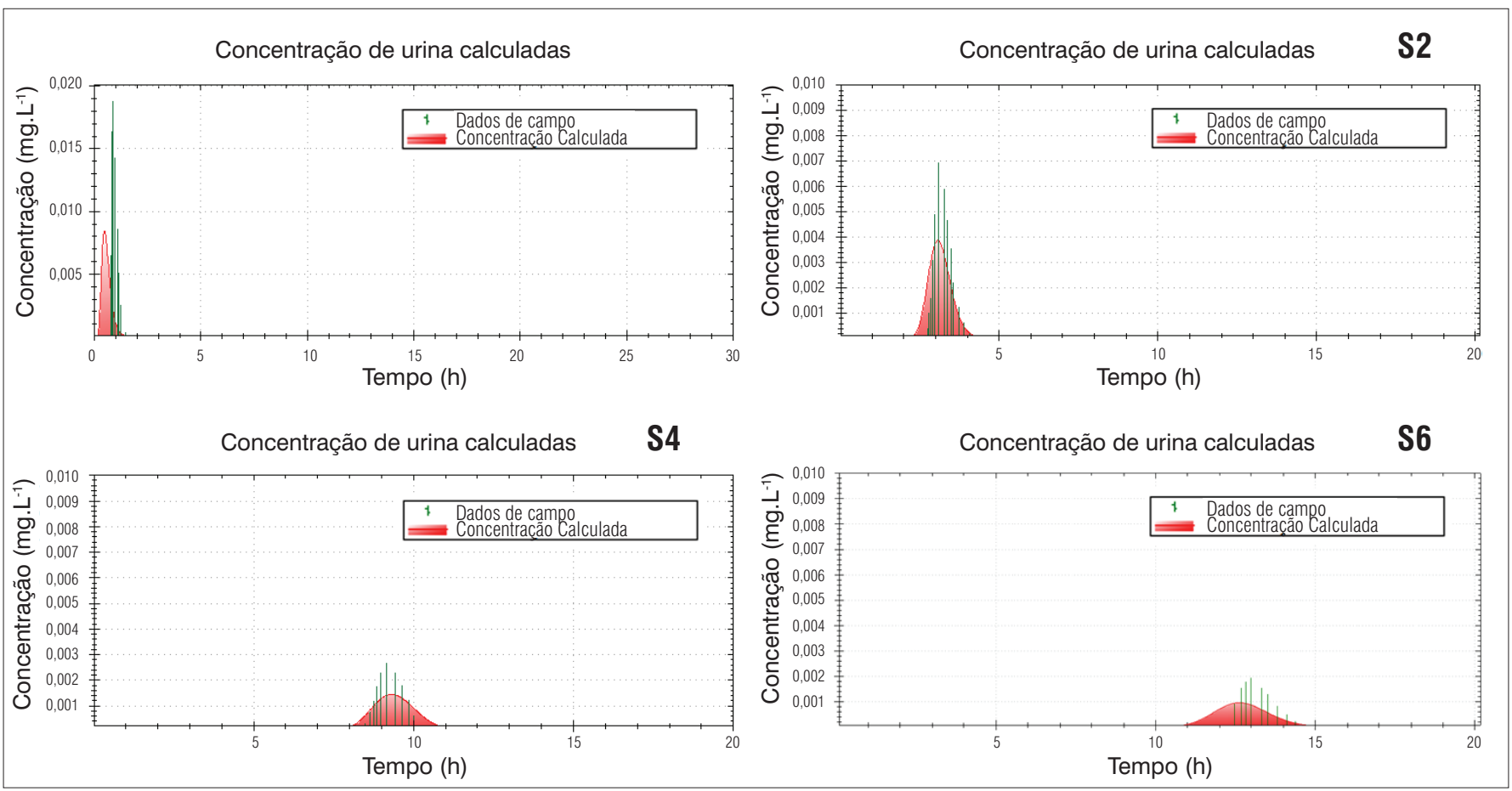

Figura 9 - Resultados da reprodução do lançamento de 5 kg de uranina no ponto So, realizado em 08 de janeiro de 1987 (RIG0, 1992). 
Tabela 2 - Resumo das diferenças entre as concentrações de pico e tempos de pico verificadas entre as plumas observadas e as simuladas para os pontos com dados observados.

\begin{tabular}{|c|c|c|c|c|c|c|}
\hline Lançamento & Vazão $\left(m^{3} \cdot s^{-1}\right)$ & Ponto & $\begin{array}{l}\text { Diferença conc. } \\
\text { de pico }\left(\mu \mathrm{g} \cdot \mathrm{L}^{-1}\right)\end{array}$ & $\begin{array}{l}\text { Erro conc. de } \\
\text { pico }(\%)\end{array}$ & $\begin{array}{l}\text { Diferença tempo } \\
\text { de pico }(\mathrm{h})\end{array}$ & $\begin{array}{l}\text { Erro tempo de } \\
\quad \text { pico (\%) }\end{array}$ \\
\hline \multirow{4}{*}{10 kg; s0; 27/09/86 } & \multirow{4}{*}{272} & $\mathrm{~S} 2$ & $-7,5$ & -58 & 0,3 & 9 \\
\hline & & S3 & $-0,6$ & -15 & 1,1 & 13 \\
\hline & & S4 & $-0,4$ & -16 & 1,2 & 10 \\
\hline & & S6 & $-0,5$ & -23 & 2,9 & 17 \\
\hline \multirow{2}{*}{5 kg; S0; 08/01/87 } & \multirow{2}{*}{342} & S2 & $-2,0$ & -29 & 0,1 & 5 \\
\hline & & S6 & $-1,0$ & -50 & 0,4 & 3 \\
\hline \multirow{3}{*}{5 kg; S0; 29/01/87 } & \multirow{3}{*}{639} & $\mathrm{~S} 2$ & $-0,6$ & -11 & 0,3 & 10 \\
\hline & & S4 & $-0,6$ & -25 & 0,1 & 2 \\
\hline & & S6 & $-0,8$ & -41 & 0,6 & 5 \\
\hline 5 kg; S0; 15/12/87 & 294 & S6 & $-1,1$ & -50 & 1,2 & 9 \\
\hline
\end{tabular}

conc.: concentração.

calculados por meio da diferença entre o valor calculado e o valor observado, esta dividida pelo valor observado e multiplicada por cem.

De uma forma geral, pode-se afirmar que as plumas simuladas utilizando o SIAQUA-IPH apresentaram um pequeno atraso na chegada do pico e uma dispersão maior que a observada em campo, resultando em polutogramas mais amortecidos, com menor concentração de pico. A diferença média entre a concentração de pico calculada e observada foi de $35 \%$, e a defasagem no tempo de pico em torno de 40 minutos. Essas diferenças podem estar relacionadas com o fato de as velocidades calculadas inicialmente estarem um pouco superestimadas e com o uso de um coeficiente de dispersão longitudinal estimado muito elevado.

Como o modelo foi desenvolvido para ser aplicável em condições de escassez de dados, os resultados gerados são limitados por erros como esses encontrados nesta aplicação no Rio Paraíba do Sul. Essa é uma limitação deste tipo de abordagem. Contudo, os resultados são aproximações das plumas observadas que são úteis para a tomada de decisão sobre o controle da qualidade da água impactada.

\section{Conclusões}

O presente trabalho apresentou o desenvolvimento um modelo de qualidade da água acoplado com um SIG denominado de SIAQUA-IPH. A concepção matemática do modelo consiste no uso de soluções analíticas unidimensionais com um esquema de convolução de polutogramas, que permite a geração de resultados rápidos com baixo custo computacional.

A integração com SIG desenvolvida possui dois aspectos positivos. O primeiro é facilitar a preparação de dados de entrada espaciais das regiões simuladas, que podem ser obtidos de forma automática usando ferramentas disponíveis de geoprocessamento. O segundo aspecto positivo é que o acoplamento com a plataforma MapWindow GIS $^{\circledR}$, de uso livre, amplia a funcionalidade e a aplicabilidade do modelo no sentido de simplificar a inserção de informações de lançamentos e a interpretação de resultados, favorecendo o entendimento sistêmico das regiões simuladas.

Além disso, para estimar os valores de parâmetros físicos, adotou-se no modelo uma série de métodos, incluindo a automatização de uma grande quantidade de equações empíricas para a estimativa do coeficiente de dispersão longitudinal visando diminuir a incerteza gerada pela escolha da equação por diferentes usuários, e formas de extrapolação de variáveis hidráulicas, como uso de equações geomorfológicas.

Para a verificação do funcionamento do modelo, das técnicas adotadas, e da implementação computacional desenvolvida, foi proposta a realização de testes de representação de dados de um ensaio com traçadores no Rio Paraíba do Sul (RJ). Os testes evidenciaram um desempenho adequado das funções de interação com um usuário e acoplamento com SIG usadas do modelo, bem como mostraram um exemplo do desempenho das demais soluções adotadas. No ensaio de representação de dados de traçadores o modelo representou com boa aproximação as plumas observadas, com um pequeno atraso na chegada do pico e uma dispersão maior que a observada em campo.

Finalmente, em modelos de qualidade da água desenvolvidos para representar lançamentos diversos, inclusive acidentais, é necessário que os resultados sejam gerados rapidamente, pois em problemas deste tipo é necessário responder em um pequeno intervalo de tempo sobre a necessidade de interromper a captação de água em 
pontos localizados a jusante do local de derramamento. Apesar de esse não ser o único uso pretendido para o SIAQUA-IPH, acredita-se que ele pode ser uma ferramenta muito útil para estes casos, dando apoio à decisão para membros de equipes técnicas de órgãos ambientais e de empresas de saneamento que têm a responsabilidade de lidar com este tipo de questão.

Outra forma de aplicação do modelo poderia ser para o estudo prévio de uma bacia, gerando cenários para diferentes vazões, para os principais pontos de interesse de lançamento e observação, o que formaria um banco de dados para consulta rápida em caso de acidentes. Nestes casos, dados de estudos ambientais com traçadores e de acidentes já ocorridos, se bem monitorados, poderiam ser usados para calibrar o modelo para um determinado trecho do rio, ou para determinar a faixa de incerteza esperada na aplicação do SIAQUA-IPH.

\section{Agradecimentos}

O primeiro autor do trabalho agradece a bolsa de estudos concedida pelo CNPq, que possibilitou o desenvolvimento deste trabalho. Os autores também agradecem aos revisores anônimos deste artigo, pelas sugestões de melhorias indicadas durante o processo de revisão.

\section{Referências}

AMES, D.P.C.; MICHAELIS, A.; ANSELMO, L.; CHEN, H.; DUNSFORD H. (2008) MapWindow GIS. In: Encyclopedia of GIS. New York: Springer. p. $633-634$

BENEDINI, M. (2011) Water Quality Models for Rivers and Streams. State of the Art and Future Perspectives. European Water, v. 34, p. 27-40.

BOWIE, G.L; MILLS, W.B.; PORCELLA, D.B.; CAMPBELL, C.L; PAGENKOPF, J.R; RUPP, G.L.; JOHNSON, K.M.; CHAN, P.W.H.; GHERINI, S.A; CHAMBERLAIN, C. (1985) Rates, constants, and kinetics formulations in surface water quality modeling EPA/600/3-85/040. 2. ed. U.S. Environmental Protection Agency, Athens, Ga. 455 p.

BUARQUE, D.C.; FAN, F.M.; PAZ, A.R.; COLLISCHONN, W. (2009) Comparação de métodos para definir direções de escoamento a partir de modelos digitais de elevação. Revista Brasileira de Recursos Hídricos, v. 14, p. 91-103.

CHANSON, H. (2004) Environmental hydraulics of open channel flows. London: Butterworth-Heinemann. 483 p.

CHAPRA, S.C. (1997) Surface water-quality modeling. New York: McGraw-Hill, Inc. 844 p

COLLISCHONN, W. (2001) Simulação Hidrológica de Grandes Bacias. Tese (Doutorado em Recursos Hídricos e Saneamento Ambiental). Instituto de Pesquisas Hidráulicas, Universidade Federal do Rio Grande do Sul, Porto Alegre, $270 \mathrm{p}$

COLLISCHONN, W. \& TUCCI, C.E.M. (2001) Simulação hidrológica de grandes bacias. Revista Brasileira de Recursos Hídricos, v. 6, n. 2, p. 95-118.

COX, B.A. (2003) A review of currently available in-stream water quality models and their applicability for simulating dissolved oxygen in lowland rivers. The Science of Total Environment Journal, v. 314, n. 316, p. 335-377.
CUNHA, A.C.; CUNHA, H.F.A.; PINHEIRO, L.A.R. (2013) Modelagem e simulação do escoamento e dispersão sazonais de agentes passivos no rio Araguari - AP: Cenários para o AHE Ferreira Gomes I - Amapá/ Brasil. Revista Brasileira de Recursos Hídricos, v. 18, n. 1, p. 34-51.

DEVENS, J.A. \& BARBOSA JÚNIOR, A.R. (2010) Modelos Empíricos e Semi-Empíricos para a Obtenção do Coeficiente de Dispersão Longitudinal de Pequenos Cursos de Água. Revista Brasileira de Recursos Hídricos, v. 15, p. 75-88

DEVENS, J.A.; BARBOSA JÚNIOR, A.R.; SILVA, G.Q. (2006) Modelo de quantificação do coeficiente de dispersão longitudinal de pequenos cursos de água naturais. Revista Engenharia Sanitária e Ambiental, v. 11, n. 3, p. 269-276.

FAN, F.M. (2011) Acoplamento entre SIG e modelos hidrológicos: Integração do modelo MGB-IPH. Trabalho de Graduação em Engenharia Ambiental. Universidade Federal do Rio Grande do Sul, Porto Alegre, $241 \mathrm{p}$.

FAN, F.M.; COLLISCHONN, W.; SORRIBAS, M.V.; PONTES, P.R.M (2013) Sobre o início da rede de drenagem definida a partir dos modelos digitais de elevação. Revista Brasileira de Recursos Hídricos (no prelo).

FERREIRA, D.M. \& CUNHA, C. (2013) Simulação numérica do comportamento térmico do reservatório do Rio Verde. Revista Engenharia Sanitária e Ambiental, v. 18, n. 1, p. 83-93.

FERRER, J.; PÉREZ-MARTíN, M.A.; JIMÉNEZ, S.; ESTRELA, T.; ANDREU, J. (2012) GIS-based models for water quantity and quality assessment in the Júcar River Basin, Spain, including climate change effects. Science of the Total Environment, v. 440, p. 42-59.

FOSTER, J.A. \& MCDONALD, A.T. (2000) Assessing pollution risks to water supply intakes using geographical information systems (GIS). Environmental Modelling \& Software, v. 15, n. 3, p. 225-234. 
GONÇALVES, J.B.; ALMEIDA, J.R.; LINS, G.A. (2007) Uma análise crítica do acidente em Cataguases-MG, 2003. Revista Ciências do Ambiente On-Line, v. 3, n. 2, 9 p.

HARARI, J.; FERREIRA, F.R.; DEGASPARI, F.A.; SARTOR, S.M. (2013) Modelagem numérica da hidrodinâmica e da dispersão de esgoto na Baía de Santos, SP. Revista Brasileira de Recursos Hídricos, v. 18 n. 1, p. 205-214.

HESSION, C.W. \& SHANHOLTZ, V.O. (1988) A geographic information system for targeting nonpoint-source agricultural pollution. Journal of Soil and Water Conservation, v. 43, n. 3, p. 264-266.

JOÃO, E.M. \& WALSH, S.J. (1992) GIS implications for hydrologic modeling: simulation of nonpoint pollution generated as a consequence of watershed development scenarios. Computers, Environment and Urban Systems, v. 16, n. 1, p. 43-63.

KILPATRICK, F.A. \& COBB, E.D. (1985) Measurement of discharge using tracers: U.S. Geological Survey Techniques of Water-Resources Investigations. Denver, EUA: U.S. Geological Survey. Report. Book 3, Cap. A16, 52 p.

LINS, R.C.; MENDES, C.A.B.; AGRA, S.G.; CARVALHO, A.E.; FRAGOSO JR, C.R. (2012) Integração de um modelo hidrológico a um SIG para avaliação da qualidade da água na Bacia do Rio Doce. Revista Brasileira de Recursos Hídricos, v. 17, p. 171-181.

MAIDMENT, D. (2002) Arc Hydro - GIS for water resources. Redlands, CA: ESRI Press. 220 p.

MARIANO, A.P.; SILVA, A.J.; OLIVEIRA, V.J.A.; ANGELIS, D.F. (2010) Avaliação da dispersão de efluente líquido de refinaria de petróleo. Engenharia Sanitária e Ambiental, v. 15, n. 3, p. 251-256.

OLIVIERI, L.J.; SCHAAL, G.M.; LOGAN, T.J.; ELLIOT, W.J.E.; MOTCH, B. (1991) Generating AGNPS input using remote sensing and GIS. Paper 91-2622. In: American Society of Agricultural Engineers Meeting, St. Joseph, Michigan. $15 \mathrm{p}$.

O'LOUGHLIN, E.M. \& BOWMER, K.H. (1975) Dilution and decay of aquatic herbicides in flowing channels. Journal of Hydrology, v. 26, n. 3-4, p. 217-235.

PAIVA, R.C.D. (2009) Modelagem hidrológica e hidrodinâmica de grandes bacias: Estudo de caso na bacia do rio Solimões. Dissertação (Mestrado em Recursos Hídricos e Saneamento Ambiental). Universidade Federal do Rio Grande do Sul, Porto Alegre, 182 p.
PAZ, A.R. \& COLLISCHONN, W. (2008) Derivação de rede de drenagem a partir de dados do SRTM. Revista Geográfica Acadêmica, v. 2, p. 84-95.

RIBEIRO, C.B.M.; SILVA, D.D.; SOARES, J.H.P.; GUEDES, H.A.S. (2011) Warning system based on theoretical-experimental study of dispersion of soluble pollutants in rivers. Engenharia Agrícola [online], v. 31, n. 5, p. 985-997.

RIGO, D. (1992) Dispersão longitudinal em rios: desempenho de métodos de previsão e de traçadores fluorescentes. Dissertação (Mestrado em Engenharia Civil). Programa de Pós-Graduação em Engenharia, Universidade Federal do Rio de Janeiro, Rio de Janeiro.

RUNKEL, R.L. (1996) Solution of the advection-dispersion equation: continuous loads of finite duration. Journal of Environmental Engineering, v. 122 , p. $830-832$.

SRINIVASAN, R. \& ARNOLD, J.G. (1994) Integration of a basin-scale water quality model with GIS. Water Resources Bulletin, v. 30, p. 453-462.

STRAGER, M.P.; FLETCHER, J.J.; STRAGER, J.M.; YUILL, C.B.; ELI, R.N.; PETTY, J.T.; LAMONT, S.J. (2010) Watershed analysis with GIS: The watershed characterization and modeling system software application. Computers \& Geosciences, v. 36, p. 970-976.

TARBOTON, D.G. (2002) Terrain Analysis Using Digital Elevation Models (Taudem). Utah Water Research Laboratory, Utah State University, Logan. Disponível em: <http://www.engineering.usu.edu/dtarb>. Acesso em: 10 maio 2013.

TIM, U.S.; MOSTAGHIMI, S.; SHANHOLTZ, V.O. (1992) Identification of critical nonpoint pollution source areas using geographic information systems and water quality modeling. Water Resources Bulletin, v. 28, p. 877-887.

USACE - United States Army Corps of Engineers. (2003) Geospatial hydrologic modeling extension: HEC-GeoHMS user's manual. Davis, CA: U.S. Army Corps of Engineers, Hydrologic Engineering Center, Report CPD-77. 197 p.

USEPA - United States Environmental Protection Agency. (2003) QUAL2E Windows Interface User's Guide. United States Environmental Protection Agency, Office of Water, Athens, EPA/823/B/95/003, Athens GA. 68 p. 